\title{
AVALIAÇÃO CLÍNICA DA INFILTRAÇÃO FACETARIA NO TRATAMENTO DA DOR LOMBAR CRÔNICA POR SÍNDROME FACETARIA - ESTUDO PROSPECTIVO
}

\author{
CLINICAL EVALUATION OF THE LUMBAR FACET JOINT BLOCKS IN TREATMENT OF \\ CHRONIC LOW BACK PAIN - A PROSPECTIVE STUDY \\ EVALUACIÓN CLÍNICA DE LA INFILTRACIÓN DE LAS FACETAS EN EL TRATAMIENTO DEL \\ DOLOR CRÓNICO DE ESPALDA BAJA POR EL SÍNDROME DE FACETA SÍNDROME - \\ ESTUDIO PROSPECTIVO
}

\author{
Thiago Miller Santana Silva", Bartolomeu ribeiro Coutinho Filho', Carlos Eduardo Gonçales Barsotti ${ }^{1}$, Francisco Prado Eugênio dos Santos², \\ Jefferson Alves Galves², Carlos Eduardo Algavez Soares de Oliveira ${ }^{3}$
}

\begin{abstract}
RESUMO
Objetivo: Avaliar os resultados do bloqueio facetário lombar com anestésico local e corticóide em pacientes com lombalgia crônica por síndrome facetária. Métodos: Realizou-se um estudo prospectivo em 30 pacientes com lombalgia crônica por síndrome facetária, os quais foram submetidos à infiltração facetária com bupivacaína a 0,25\% e acetato de metilpredinisolona sob controle radioscópico, e foram seguidos e avaliados seguindo as escalas Visual Analógica da Dor, Oswestry Disability Index e os Critérios de MacNab nos quais 17 foram do sexo feminino e 13 do sexo masculino. Resultados: Foi observada diminuição significativa $(p<0,05)$ da dor pela Escala Visual Analógica. Obtivemos 73,3\% de resultados satisfatórios na primeira semana, 63,3\% no primeiro mês, 43,3\% em 3 meses, e 36,6\% no final de 6 meses. O índice de complicações foi muito baixo. Conclusão: O bloqueio facetário utilizando corticóide e anestésico local se mostrou-se efetivo, seguro e de fácil realização. No entanto houve recidiva em mais de 50\% dos casos dentro de 6 meses.
\end{abstract}

Descritores: Lombalgia; Articulação zigoapofisaria, Bloqueio nervoso.

\begin{abstract}
Objective: To evaluate the lumbar facet block with local anesthetics and corticosteroids in patients with chronic low back pain of facet joint origin. Methods: We conducted a prospective study on 30 patients with chronic low back pain in facet syndrome who underwent facet infiltration with bupivacaine $0.25 \%$ and methylprednisolone acetate under radioscopic control; the subjects were followed and evaluated following the Visual Analogue Scale of Pain, Oswestry Disability Index and MacNab criteria; 17 of them were female and 13 male. Results: Reduction of pain was found by Visual Analogue Scale significant ( $p<0.05)$. We obtained $73.3 \%$ of satisfactory results in the first week, $63.3 \%$ in the first month, $43.3 \%$ in third months, and $36.6 \%$ at the end of 6 months. The complication rate was very low. Conclusion: Facet blocka using local anesthetic and steroid was effective, safe and easy to perform. Neverthelees, there was recurrence of most cases within six months.
\end{abstract}

Keywords: Low back pain; Zigapophyeal joints; Nérvea block.

\section{RESUMEN}

Objetivo: Evaluar los resultados del bloqueo facetario lumbar, con anestésico local y corticoide, en pacientes con dorsalgia crónica por síndrome facetario. Métodos: Se realizó un estudio prospectivo en 30 pacientes, 17 del sexo femenino y 13 del sexo masculino, con dorsalgia crónica por síndrome facetario, quienes fueron sometidos a la infiltración facetaria con bupivacaína a 0,25\% y acetato de metilpredinisolona bajo control radioscópico, y fueron acompañados y evaluados siguiendo las escalas Visual Analógica del Dolor, Oswestry Disability Index y los Criterios de MacNab. Resultados: Se observó una disminución significativa $(p<0,05)$ del dolor según la Escala Visual Analógica del Dolor. Obtuvimos 73,3\% de resultados satisfactorios en la primera semana, 63,3\% en el primer mes, 43,3\% en 3 meses, y 36,6\% al final de 6 meses. El índice de complicaciones fue muy bajo. Conclusión: El bloqueo facetario, utilizando corticoide y anestésico local, se mostró eficaz, seguro y de fácil realización, no obstante, con recidiva en la mayor parte de los casos dentro de 6 meses.

Descriptores: Dolor en la región lumbar; Articulación Cigapofisaria; Metilprednisolona; Estudios prospectivos.

\section{INTRODUÇÃO}

A dor lombar crônica é um problema de grande importância na sociedade, resultando em um grande número de consultas médicas e a um prejuizo enorme em faltas ao trabalho ${ }^{1}$. Existem várias causas que podem levar a dor lombar crônica, estando os discos intervertebrais, as articulações facetárias e a articulação sacroiliaca entre as principais. A articulação facetária tem sido implicada como a fonte causadora de lombalgia em cerca de 15 a $45 \%$ dos casos de dor lombar crônica ${ }^{2-3}$, baseado na resposta a testes com bloqueio diagnostico de acordo com critérios estabelecidos pela International Association for the Study of Pain 4 .

A articulação facetária é uma articulação sinovial, envolvida por uma cápsula ricamente vascularizada e inervada ${ }^{5}$ pelo ramo medial do ramo dorsal do nervo espinhal ${ }^{6,7}$, que vem sendo suspeitada

1. Medico Colaborador de Cirurgia da Coluna do Hospital do Servidor Publico Estadual - São Paulo, SP, Brasil.
2. Medico Assistente de Cirurgia da Coluna do Hospital do Servidor Publico Estadual - São Paulo, SP, Brasil .

3. Mestre em Ortopedia do Hospital do Servidor Público Estadual - São Paulo, SP, Brasil.

Trabalho realizado no Hospital do Servidor Publico Estadual - HSPE. São Paulo, SP, Brasil.

Correspondência: Rua Borges Lagoa, 1755, Sala 180, - Vila Clementino - CEP.: 04038-034 - São Paulo,SP. Brasil. Email: tmillergv@hotmail.com

Recebido em 19/03/2011, aceito em 03/08/2011 
como fonte de dor lombar desde o inicio do século $X X^{8}$. Estudos em voluntários hígidos têm reproduzido o quadro de dor lombar e dor irradiada para membros inferiores após estímulos na articulação zigoapofisaria ${ }^{9,10}$.

O diagnóico de dor lombar relacionada à síndrome facetaria é feito clinicamente junto com a exclusão de outras causas de dor lombar. O sintoma típico é a dor lombar crônica que pode ser uni ou bilateral, pode irradiar para os MMII sem um padrão radicular, geralmente não ultrapassando os joelhos ${ }^{11,12}$. O paciente normalmente refere alívio da dor com a flexão do tronco e relata piora com a extensão do tronco, movimentos rotatórios e atividades físicas ${ }^{13}$.

Vários métodos de tratamento vêm sendo propostos, desde os não invasivos como o repouso relativo, a fisioterapia, a acupuntura, os analgésicos e os corticóides ${ }^{14}$. Ate métodos mais agressivos tem sido descrito como forma de tratamento como a denervação por cirurgia aberta, a artrodese com parafuso pedicular, a fixação traslaminar e a facetária ${ }^{14,15}$. Que podem por sua vez trazer muitas complicações associadas. Atualmente muitas técnicas minimamente invasivas vêm ganhando espaço e se demonstrando efetivas no tratamento da dor lombar crônica, como é o caso da infiltração intra articular ${ }^{16,17}$, do bloqueio do ramo nervoso que supre a articulação facetária ${ }^{18,19}$, e a neurotomia por radiofreqüência. A técnica de bloqueio da inervação da faceta com corticóide e anestésico local vem sendo utilizada há muitos anos e modificada de várias formas ${ }^{19}$. Apesar de a técnica ser largamente utilizada, poucos estudos randomizados e bem controlados tem sido relatados na literatura ${ }^{16,20}$, o que vem trazendo controvérsias a respeito da real efetividade desta modalidade de tratamento.

O presente estudo procurou estudar a técnica de bloqueio facetário com anestésico local e corticóide, visando definir a eficácia do método, a duração do período de alivio da dor e a segurança.

\section{MÉTODOS}

O projeto foi enviado e aprovado pela Comissao de ensino e pesquisa (CET) do Hospital do Servidor Publico Estadual de Sao Paulo. No período de julho de 2009 a julho de 2010, quarenta e dois pacientes com suspeita clínica de síndrome facetária foram submetidos ao bloqueio anestésico diagnóstico, em sala cirurgica sob fluoroscopia intra operatoria, com $0,5 \mathrm{ml}$ de lidocaína a $1 \%$, sendo confirmado o diagnostico em 30 desses pacientes. Destes 17 eram do sexo feminino e 13 do sexo masculino. A idade variou de 33 a 78 anos (média de 57,4 anos). O diagnóstico da síndrome facetária foi feito por um cirurgião de coluna experiente, através da avaliação clínica dos pacientes que apresentavam dor lombar crônica com piora a extensão do tronco, e complementado em todos os casos com estudo por ressonância magnética evidenciando a presença de sinais de artrite facetáia, e excluindo outros possíveis diagnósticos. Esses pacientes foram submetidos a um teste diagnostico inicial com lidocaína a 1\%. Aqueles que demonstraram alívio da dor em ate $4 \mathrm{~h}$ apos bloqueio facetario , foram incluídos no estudo e foram considerados portadores de síndrome facetária.

Como critérios de inclusão foram considerados pacientes que apresentassem dor lombar crônica há no mínimo seis meses, com piora pela manhã e a extensão do tronco, e que apresentaram um teste diagnostico positivo com lidocaína a 1\%. Como critérios de exclusão foram considerados pacientes que apresentavam déficit neurológico, doença reumatológica, coagulopatia, gravidez, alergia a anestésico local, incompatibilidade com corticosteróide, cirurgia na coluna lombar prévia, lesão tumoral, espondilolistes ou outra causa que pudesse justificar a dor.

Foi utilizado técnica padrão por um cirurgião de coluna experiente, em que o paciente foi posicionado em decúbito ventral em mesa radiotransparente no centro cirúrgico, feito o preparo da pele com solução degermante. Utiliza-se radioscopia para marcação e posicionamento da agulha, é feito o bloqueio de pele com lidocaína a 1\%, em seguida é feito o bloqueio do ramo medial do ramo posterior do nervo espinhal que inerva a faceta articular com uma solução de 0,5 ml de bupivacaina a 0,25\% e 0,5 ml (20mg) de acetato de metil predinisolona utilizando-se agulha de 22 gauge. O bloqueio foi feito no mínimo em dois níveis, sendo que somente foram inclusos no estudo bloqueios nos niveis L3-4, L4-5 e L5-S1, unilateral ou bilateral, dependendo do exame físico e da resposta ao teste do bloqueio diagnóstico.

Para avaliação dos resultados foram utilizados a Escala Visual Analógica (EVA), avaliação funcional Oswestry Disabity Index 2.0 (ODI) e os critérios de MacNab. A Escala Visual Analógica é graduada de 0 a 10 em que o menor valor equivale a ausência de dor e o maior valor equivale a dor de mais forte intensidade. $\mathrm{Na}$ avaliação funcional Oswestry Disabity Index 2.0 (ODI), o paciente emite uma pontuação referente a uma serie de atividades do cotidiano como presença de dor, cuidados pessoais, capacidade de elevar peso e sono. Já na avaliação pelos critérios de MacNab o resultado do paciente é graduado após o procedimento como excelente, bom, regular e ruim quanto a resposta de satisfação do paciente. Os pacientes foram avaliados antes da infiltração e após o período um dia, uma semana, um mês, três meses e seis meses. Foi obtida a devida aprovação da comissão de ética da instituição e não houve conflito de interesses. Foram realizados para análise estatísticas os testes $t$ de student para variáveis continuas e o teste do Qui-Quadrado para variáveis dicotômicas através do software SPSS para Windows. Foi utilizado como significância estatistica um $\mathrm{p}<0,05$.

\section{RESULTADOS}

Pela avaliação com a Escala Visual Analógica da dor houve uma queda significativa da dor $(p<0,05)$ no dia seguinte e nas semanas consecutivas ao procedimento, apesar da tendência a retornar aos padrões inicias ao final de seis meses ( Figura 1).

A Figura 2 demonstra os resultados de acordo com os critérios de MacNab. Considerado-se como resultados satisfatórios os casos que tiveram bom ou excelente resultado. Obtivemos 73,3\% de resultados satisfatórios na primeira semana, 63,3\% de resultados satisfatórios no primeiro mês, $43,3 \%$ de resultados satisfatórios em três meses, e $36,6 \%$ de resultados satisfatórios no final de seis meses.

Pela avaliação funcional pelo Oswestry Disability Index 2.0 (Figura 3) encontramos uma queda de 47,6\% do valor pré tratamento em três meses e uma queda de apenas $11 \%$ após 6 meses em relação aos valores pré tratamento.

Não foram observado complicações importantes relacionadas ao procedimento, com apenas três casos que referiram parestesia no trajeto nervoso relacionado a infiltração por poucas horas.

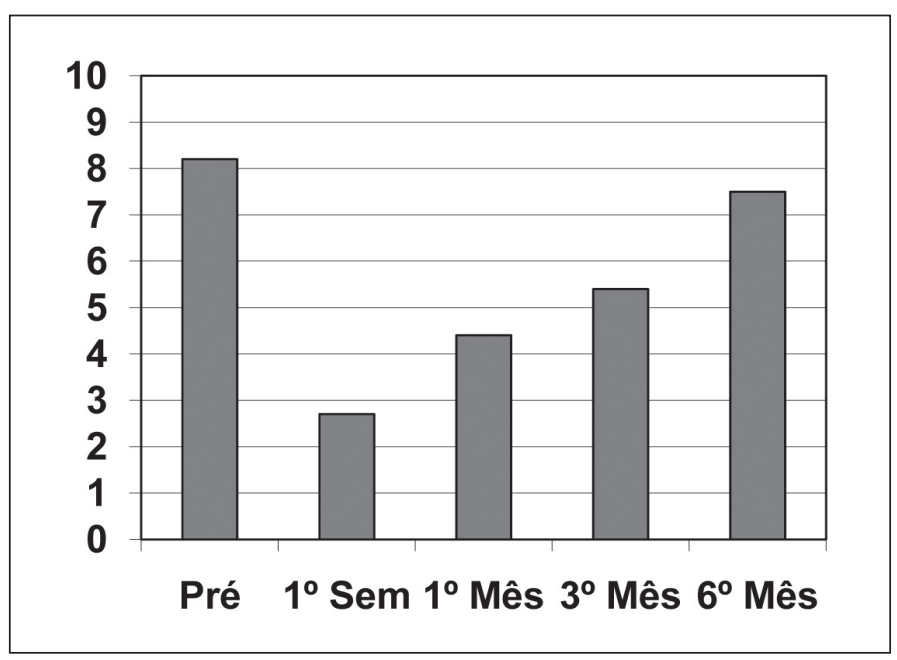

Figura 1. Resultados obtidos através da escala analógica da dor e apresentados antes do procedimento (Pré), após o período de primeiro semestre, um mês, três meses e seis meses $(p<0,05)$. 


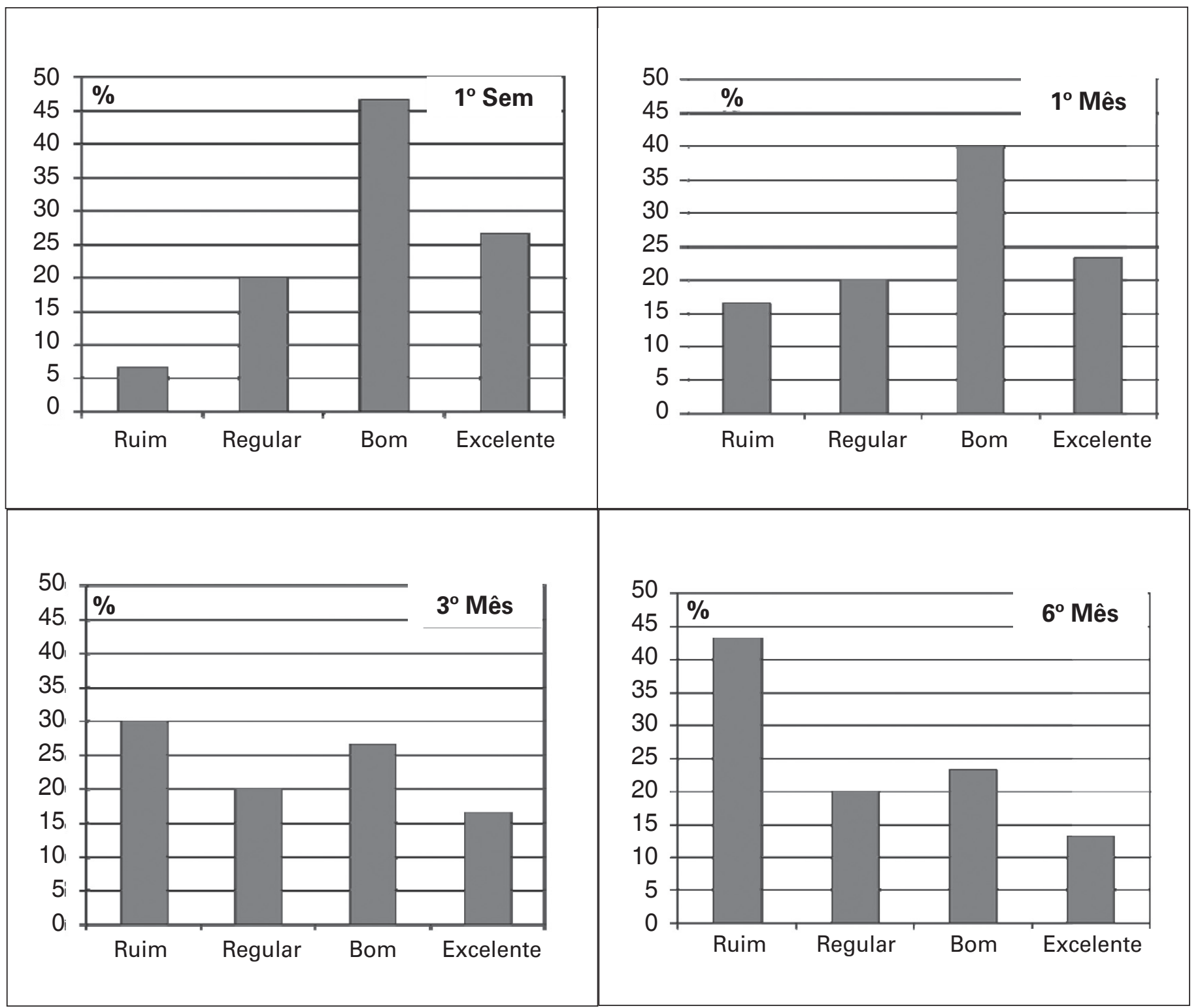

Figura 2. Resultados obtidos utilizando-se os critérios de MacNab após primeiro semestre, um mês, três meses e seis meses.

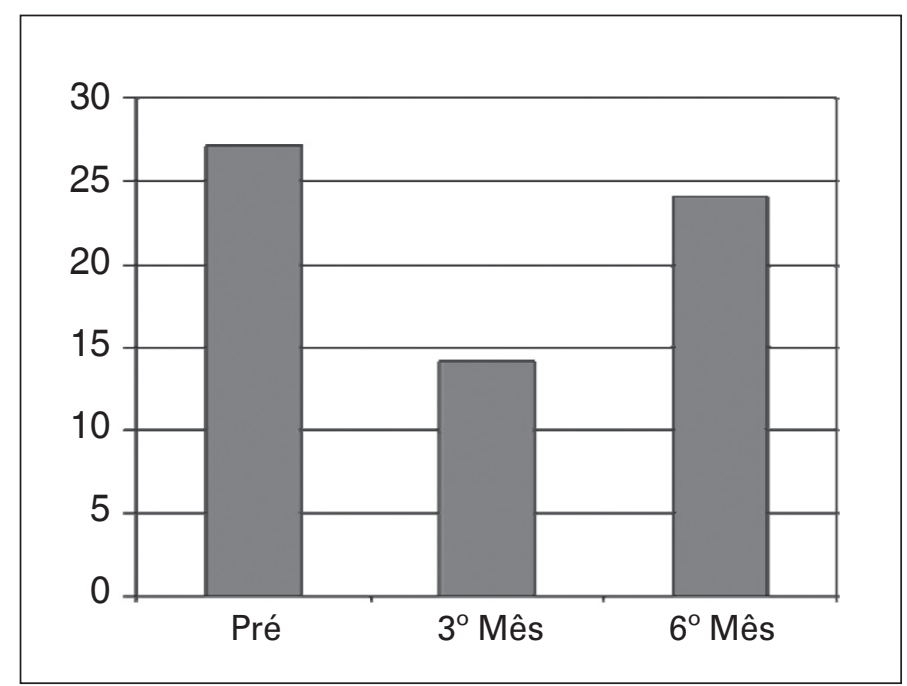

Figura 3. Resultados obtidos através do Oswestry Disability Index 2.0 antes do procedimento (Pré), com três meses e seis meses.

\section{DISCUSSÃO}

Existem na literatura diversos trabalhos sobre o tratamento da síndrome facetária, apresentando uma grande diversidade de resultados favoráveis e desfavoráveis ${ }^{3,5-7}$, o que deixa o tema ainda em discussão sobre a real eficácia de tais tratamentos ${ }^{7,8}$. O propósito deste estudo foi tentar elucidar melhor esta questão, definindo principalmente a eficácia do tratamento feito com anestésico local e corticosteróide e o tempo médio livre de dor dos pacientes submetidos a um único tratamento

Uma crítica a este estudo seria a amostra que não foi muito extensa e o fato de o estudo não ter grupo controle e não ser randomizado, o que predispõe a existência de viés.

Nosso estudo sugere que a técnica seja eficaz por um período limitado de tempo na maior parte dos pacientes, com uma tendência a retornar aos padrões inicias passados três meses, como ja havia sido observado em outros trabalhos na literatura ${ }^{11,12}$, porém pela pequena morbidade da técnica o procedimento pode ser repetido para se obter resultados mais duradouros ${ }^{16}$.

O tratamento proposto neste estudo se trata de um procedimento minimamente invasivo o que explica o baixo número de 
complicações encontradas, como foi o caso dos três pacientes que apresentaram parestesia no membro inferior correspondente a infiltração o que se correlacionaria ao contato do anestésico local com a raiz nervosa adjacente.

Existem na literatura vários protocolos para infiltração facetária utilizando desde anestésico local , corticóide, álcool,fenol entre outros, isoladamente ou em uma combinação entre eles ${ }^{16,18-20}$. No presente estudo foi utilizado um anestésico local, que possivelmente teria um efeito de romper o ciclo vicioso de dor presente nestes pacientes ${ }^{7}$, e um corticóide de depósito que teria um efeito antiinflamatório a curto e a longo prazo no local. Por outro lado tais substâncias são largamente utilizadas , o que nos garante mais segurança na utilização neste protocolo.

Atualmente uma série de formas alternativas estão sendo estu- dadas com o intuito de definir qual seria o melhor tratamento para estes pacientes com lombalgia crônica por síndrome facetária ${ }^{12,16,20}$, como é o caso da radiofreqüência que atualmente vem sendo motivo de vários estudos dos quais tem se demontrado resultados variáveis $^{21,22}$. No presente momento ainda se faz necessário mais estudos prospectivos, randomizados, placebo controlado bem conduzido para definir qual método será o mais adequado para o tratamento de tais pacientes ${ }^{8}$.

\section{CONCLUSÃO}

O tratamento proposto de bloqueio facetário no presente estudo é efetivo, de fácil realização e seguro, podendo ser uma arma útil no arsenal terapêutico para o tratamento da dor lombar crônica quando indicado em casos devidamente diagnosticados.

\section{REFERÊNCIAS}

1. Scientific approach to the assessment and management of activity-related spinal disorders. A monograph for clinicians. Report of the Quebec Task Force on Spinal Disorders. Spine (Phila Pa 1976). 1987:12(7 Suppl):S1-59.

2. Manchukonda R, Manchikanti KN, Cash KA, Pampati V, Manchikanti L. Facet joint pain in chronic spinal pain: an evaluation of prevalence and false-positive rate of diagnostic blocks. J Spinal Disord Tech. 2007;20(7):539-45

3. Manchikanti L, Manchukonda R, Pampati V, Damron KS, McManus CD. Prevalence of facet joint pain in chronic low back pain in postsurgical patients by controlled comparative local anesthetic blocks. Arch Phys Med Rehabil. 2007;88(4):449-55

4. Merskey H, Bogduk N. Classification of chronic pain. In: Merskey H, Bogduk N. Classification of chronic pain descriptions of chronic pain syndromes and definition of pain terms. Itália: Intl Assn for the Study of Pain; 1994. p.180-1.

5. Shealy CN. Facet denervation in the management of back and sciatic pain. Clin Orthop Relat Res. 1976;(115):157-64.

6. Bogduk N, Long DM. The anatomy of the so-called "articular nerves" and their relationship to facet denervation in the treatment of low-back pain. J Neurosurg. 1979:51(2):172-7.

7. Bogduk N. International Spinal Injection Society guidelines for the performance of spinal injection procedures. Part 1: Zygapophysial joint blocks. Clin J Pain. 1997;13(4):285-302.

8. Abdi S, Datta S, Trescot AM, Schultz DM, Adlaka R, Atluri SL, et al. Epidural steroids in the management of chronic spinal pain: a systematic review. Pain Physician. 2007;10(1):185-212.

9. Bogduk N. Low back pain. In Bogduk's Clinical Anatomy of Lumbar Spine and Sacrum. 4th edition. New York: Churchill Livingstone; 2005. p.183-216.

10. Windsor RE, King FJ, Roman SJ, Tata NS, Cone-Sullivan LA, Thampi S, et al. Electrical stimulation induced lumbar medial branch referral patterns. Pain Physician. 2002;5(4):347-53.

11. Helbig T, Lee CK. The lumbar facet syndrome. Spine (Phila Pa 1976). 1988;13(1):61-4

12. Lippitt AB. The facet joint and its role in spine pain. Management with facet joint injections. Spine (Phila Pa 1976). 1984;9(7):746-50

13. Eisenstein SM, Parry CR. The lumbar facet arthrosis syndrome. Clinical presentation and articular surface changes. J Bone Joint Surg Br. 1987;69(1):3-7.

14. Geraci MC, Baker RM, Sasso RC. Lumbar zygapophysial joint evaluation and treatment. SpineLine. 2005:10-7.

15. Ferrara LA, Secor JL, Jin BH, Wakefield A, Inceoglu S, Benzel EC. A biomechanica comparison of facet screw fixation and pedicle screw fixation:effects of short-term and long-term repetitive cycling. Spine (Phila Pa 1976).2003;28(12):1226-34.

16. Carette S, Marcoux S, Truchon R, Grondin C, Gagnon J, AllardY, Latulippe M. A controlled trial of corticosteroid injections into facet joints for chronic low back pain. N Engl J Med. 1991:325(14):1002-7.

17. Schulte TL, Pietilä TA, Heidenreich J, Brock M, Stendel R. Injection therapy of lumbar facet syndrome: a prospective study. Acta Neurochir (Wien). 2006:148(11):1165-72.

18. Manchikanti L, Manchikanti KN, Manchukonda R, Cash KA, Damron KS, et al. Evaluation of lumbar facet joint nerve blocks in the management of chronic low back pain: preliminary report of a randomized, double-blind controlled trial: clinical trial NCT00355914. Pain Physician. 2007:10(3):425-40.

19. Manchikanti L, Manchikanti KN, Damron KS, Pampati V. Effectiveness of cervical medial branch blocks in chronic neck pain: a prospective outcome study. Pain Physician. 2004;7(2):195-201.

20. Lilius G, Laasonen EM, Myllynen P, Harilainen A, Grönlund G. Lumbar facet joint syndrome. A randomised clinical trial. J Bone Joint Surg Br. 1989;71(4):681-4.

21. van Kleef $M$, Barendse GA, Kessels A, Voets HM, Weber WE, de Lange S. Randomized trial of radiofrequency lumbar facet denervation for chronic low back pain. Spine (Phila Pa 1976). 1999;24(18):1937-42.

22. van Wijk RM, Geurts JW, Wynne HJ, Hammink E, Buskens E, Lousberg R, et al. Radiofrequency denervation of lumbar facet joints in the treatment of chronic low back pain: a randomized, double-blind, sham lesion-controlled trial. Clin J Pain. 2005;21(4):335-44. 